\title{
Atmospheric turbulence and wind profiles monitoring with generalized scidar
}

\author{
R. Avila ${ }^{1}$, J. Vernin ${ }^{2}$, and L. J. Sánchez ${ }^{3}$ \\ 1 Instituto de Astronomía UNAM, Apdo. Postal 72-3 (Xangari), 58089 Morelia Mich., México \\ 2 UMR 6525 Astrophysique, Université de Nice Sophia Antipolis/CNRS, Parc Valrose, \\ 06108 Nice Cedex 2, France \\ e-mail: Jean.VERNIN@unice.fr \\ 3 Instituto de Astronomía UNAM, Apdo. Postal 70-264, 04510 México D.F., México \\ e-mail: leonardo@astroscu.unam.mx
}

Received 28 August 2000 / Accepted 12 December 2000

\begin{abstract}
We present a method for simultaneous measurement of the vertical distribution of the optical turbulence strength $C_{\mathrm{N}}^{2}(h)$ and wind velocity $\boldsymbol{V}(h)$ in the Earth's atmosphere, based on an analysis of spatio-temporal correlations of stellar scintillation images obtained with generalized scidar. A statistical comparison of $\boldsymbol{V}(h)$ obtained with this method and instrumented balloons supports the use of this method. The algorithm used allows for the identification of dome seeing, which can be subtracted from $C_{\mathrm{N}}^{2}(h)$, to obtain a turbulence profile free of dome contribution. Examples of simultaneous $C_{\mathrm{N}}^{2}(h)$ and $\boldsymbol{V}(h)$ monitorings are presented.
\end{abstract}

Key words. atmospheric effects - turbulence - site testing - methods: data analysis - instrumentation: adaptive optics

\section{Introduction}

Knowledge of the vertical profiles of the refractiveindex structure constant $C_{\mathrm{N}}^{2}(h)$ (the optical turbulence strength) and the wind velocity $\boldsymbol{V}(h)$ is fundamental for the development and optimal operation of adaptive optics (AO) systems in optical astronomy. In addition to being essential for site characterization, these data lead to the principal atmospheric parameters related to high angular resolution imaging. The spatio-angular parameters are related to $C_{\mathrm{N}}^{2}(h)$, and the temporal parameters to $\boldsymbol{V}(h)$ and $C_{\mathrm{N}}^{2}(h)$.

For practical reasons, the most convenient method for the measurement of $C_{\mathrm{N}}^{2}(h)$ and $\boldsymbol{V}(h)$ is to determine them remotely, by optical means, which can be achieved with the scidar (an acronym of scintillation detection and ranging) technique (Rocca et al. 1974; Caccia et al. 1987; Vernin 1992). A recent improvement of this technique (Avila et al. 1997a; Fuchs et al. 1994; Fuchs 1995; Tallon 1989), leading to the generalized scidar (GS), enabled the detection of turbulence along the whole optical path, while the classical scidar was insensitive to turbulence in the first kilometer above the ground. The principle for detecting the wind velocity in the turbulent layers from the

Send offprint requests to: R. Avila,

e-mail: $r$.avila@astrosmo.unam.mx spatio-temporal correlation of scintillation images was introduced in the pioneering paper of Rocca et al. (1974). However, as they used the scintillation from a single star, they could not determine simultaneously the layer altitude and the turbulence strength. Further efforts were conducted in the same direction by Caccia et al. (1987). If one uses the scintillation of a double star instead of that of a single, it is possible to measure simultaneously $C_{\mathrm{N}}^{2}(h)$ and $\boldsymbol{V}(h)$, which is the principle used in this paper. This idea was first used by Klückers et al. (1998), after a private communication we held with these authors at the Canary Islands in 1995. Several authors have investigated the wind velocity in turbulent layers from the spatio-temporal correlation of the wavefront slopes. Gendron \& Lena (1996), Noel (1997), Schöck \& Spillar (1998) and St-Jacques \& Baldwin (2000) used Shack-Hartmann wavefront sensors for the slope measurements, while Avila et al. (1997b), Martin et al. (1998), Tokovinin et al. (1998) and Conan et al. (2000a) used the generalized seeing monitor, an instrument specially dedicated to the wavefront outer scale monitoring.

Here we present the methods and tests of the first survey of $C_{\mathrm{N}}^{2}(h)$ and $\boldsymbol{V}(h)$ along the entire optical path, using GS. The velocity profiles are statistically compared with profiles measured using instrumented balloon flights. Using a very similar method, Klückers et al. (1998) 
presented a few turbulence and wind profiles, whereas here the tests are applied to a formal survey. Also for the first time, the turbulence in the telescope dome is quantified and distinguished from the rest of the atmosphere, exploiting a recently published idea (Avila et al. 1998). In Sect. 2, the principle of the method is described, followed by the detailed algorithm. The method was applied to the data collected during 4 one-week runs in 1998 for the characterization of Cerro Pachón, Chile, which is the site of the Gemini South Telescope. The observational campaign is described in Sect. 3. The results concerning the wind profiles and the dome seeing detection are presented in Sect. 4. Finally, in Sect. 5 we give the conclusion and some consequences of this work for the development of the Gemini South AO system.

\section{Methods of measurement and analysis}

\subsection{Principle of the generalized scidar as a $C_{N}^{2}$ profiler}

The scidar technique has been extensively explained elsewhere (Rocca et al. 1974; Caccia et al. 1987; Vernin 1992). Avila et al. (1997a) presented the first experimental implementation and results of the GS, as a $C_{\mathrm{N}}^{2}$ profiler, the concept of which was introduced by Fuchs et al. (Fuchs et al. 1994; Fuchs 1995; Fuchs et al. 1998), after an original idea of Tallon (1989). For completeness, we give here a brief overview of the GS technique.

This technique consists of computing the spatial autocorrelation function of short exposure-time images of the scintillation pattern produced by a double star. In classical scidar, images of the telescope pupil are taken, which makes it insensitive to turbulence close to the ground because the scintillation variance is proportional to $h^{5 / 6}$ (Roddier 1981), where $h$ is the altitude above the ground of the turbulent layer (which acts as a phase screen) above the ground. In GS, the plane of the detector is made the conjugate of a plane at a distance $h_{\mathrm{gs}}$ (the analysis plane), which lies a few kilometers below the telescope pupil (i.e. $\left.h_{\mathrm{gs}}<0\right)$. In this case the turbulence at ground level, including that in the telescope dome, becomes detectable because the distance relevant for scintillation produced by a turbulent layer at an altitude $h$ is now $\left|h-h_{\mathrm{gs}}\right|$.

The autocorrelation of the scintillation produced by a turbulent layer consists of three components: one centered at the origin, and the two others separated by $\rho H$ and $-\boldsymbol{\rho} H$, respectively, where $\boldsymbol{\rho}$ is the angular separation of the double star and

$H=\left|h-h_{\mathrm{gs}}\right|=h-h_{\mathrm{gs}}$,

the last equality holding for the case of interest, $h_{\mathrm{gs}}<0$. As the different turbulent layers are statistically independent, the contribution of each one is added, so the total theoretical autocorrelation function can be written as:

$C^{* *}(\boldsymbol{r})=\int_{0}^{\infty} \mathrm{d} h C_{\mathrm{N}}^{2}(h)\{a \mathcal{C}(\boldsymbol{r}, H)+b[\mathcal{C}(\boldsymbol{r}-\boldsymbol{\rho} H, H)$

$$
+\mathcal{C}(\boldsymbol{r}+\boldsymbol{\rho} H, H)]\}
$$

where $\mathcal{C}(\boldsymbol{r}, H)$ represents the autocorrelation of the scintillation of a single star produced by a layer of unit $C_{\mathrm{N}}^{2}$ at a distance $H$ from the conjugated plane of the detector. The factors $a$ and $b$ are given by

$a=\frac{1+\alpha^{2}}{(1+\alpha)^{2}}, \quad b=\frac{\alpha}{(1+\alpha)^{2}}, \quad \alpha=10^{-0.4 \Delta m}$,

where $\Delta m$ is the magnitude difference of the double star.

Equation (2) shows that all the information needed to retrieve $C_{\mathrm{N}}^{2}(h)$ is contained in a radial section of $C^{* *}(\boldsymbol{r})$ along the double star separation. Furthermore, we wish to eliminate the central peak in which the contribution of each layer is undistinguishable from that of the others, as they are added, and it contains the uncorrelated noise. For this purpose we calculate the difference of the sections of the measured autocorrelation function parallel and perpendicular to the stars separation, $C_{\|}^{* *}$ and $C_{\perp}^{* *}$ respectively, which can be written as (Avila et al. 1997a):

$$
\begin{aligned}
B_{\mathrm{gs}}^{* *}(x) & \equiv C_{\|}^{* *}-C_{\perp}^{* *} \\
& =\int_{-h_{\mathrm{gs}}}^{+\infty} \mathrm{d} h K(x, h) C_{\mathrm{N}}^{2}\left(h-h_{\mathrm{gs}}\right)+N(x) .
\end{aligned}
$$

$N(x)$ is the noise, and the kernel $K(x, h)$ is a radial section of $b \mathcal{C}(\boldsymbol{r}-\boldsymbol{\rho} H, H) * S(\boldsymbol{r})$ along the direction $\boldsymbol{r} \| \boldsymbol{\rho}$, where $S(\boldsymbol{r})$ is the autocorrelation of the impulse response of the detector. By measuring $B_{\mathrm{gs}}^{* *}(x)$, calculating theoretically $K(x, h)$ and knowing $h_{\mathrm{gs}}$ as well as an estimation of the noise variance, Eq. (4) (of Fredholm type) is inverted to estimate $C_{\mathrm{N}}^{2}(h)$ using a maximum entropy algorithm. The inversion is simplified by the diagonal elements in $K(x, h)$ along the line $x=\rho\left(h-h_{\mathrm{gs}}\right)$.

Two important features of the GS should be noted: The space between the telescope pupil and the analysis plane is turbulence-free because it is virtual. The vertical resolution of the obtained $C_{\mathrm{N}}^{2}(h)$ profiles (Vernin \& Azouit 1983) is given by

$\Delta H=\frac{\mathcal{L}\left(h-h_{\mathrm{gs}}\right)}{\rho}=\frac{0.5}{\rho} \sqrt{\lambda\left(h-h_{\mathrm{gs}}\right)}$,

where $\mathcal{L}\left(h-h_{\mathrm{gs}}\right)$ is the equivalent width of the scintillation spatial autocorrelation function, related to the first Fresnel zone, and $\lambda$ is the wavelength. As an example, the resolution achieved at ground level $(h=0)$ when observing the double star 95 Herculis $(\rho=6.2 \operatorname{arcsec})$, with the analysis plane at $h_{\mathrm{gs}}=-4 \mathrm{~km}$, is $\Delta H \simeq 740 \mathrm{~m}$.

\subsection{Simultaneous measurement of $C_{N}^{2}(h)$ and $V(h)$}

\subsubsection{Principle}

The Taylor hypothesis of "frozen turbulence" postulates that turbulence is carried by wind without deformation, which is valid during short time-intervals (Schöck \& Spillar 1999). The scintillation pattern produced by a layer where the wind velocity is $\boldsymbol{V}$ will move on the analysis plane a distance $\boldsymbol{V} \Delta t$ in a time $\Delta t$. The wind velocity 
can be determined by measuring the displacement $\boldsymbol{V} \Delta t$, which can be achieved by computing the cross-correlation of scintillation images taken at times separated by a known constant delay $\Delta t$. When the source is a double star, the cross-correlation will result in three peaks (that we call a triplet), as in the case of the autocorrelation, but the central peak will no longer be situated at the origin but at the point $\boldsymbol{r}=\boldsymbol{V} \Delta t$. As in the autocorrelation, the separation between the central and the lateral peaks is $\boldsymbol{\rho} H$ and $-\boldsymbol{\rho} H$ (see Sect. 2.1). In the realistic case of multiple layers, by analogy to Eq. (2), the cross-correlation is written as:

$$
\begin{array}{rl}
C_{\mathrm{c}}^{* *}(\boldsymbol{r}, \Delta t)=\int_{0}^{\infty} \mathrm{d} & h C_{\mathrm{N}}^{2}(h)\left\{a \mathcal{C}_{\mathrm{c}}(\boldsymbol{r}-\boldsymbol{V}(h) \Delta t, H)\right. \\
& +b\left[\mathcal{C}_{\mathrm{c}}(\boldsymbol{r}-\boldsymbol{V}(h) \Delta t-\boldsymbol{\rho} H, H)\right. \\
& \left.\left.+\mathcal{C}_{\mathrm{c}}(\boldsymbol{r}-\boldsymbol{V}(h) \Delta t+\boldsymbol{\rho} H, H)\right]\right\},
\end{array}
$$

$H$ being defined by Eq. (1). $\mathcal{C}_{\mathrm{c}}$ differs from $\mathcal{C}$ (Eq. 2) only by an eventual temporal decorrelation of the scintillation (partial failure of the Taylor hypothesis) and an eventual fluctuation of $\boldsymbol{V}(h)$ during the integration time. The decorrelation would make $\mathcal{C}_{\mathrm{c}}$ smaller than $\mathcal{C}$, and the fluctuation of $\boldsymbol{V}(h)$ would make $\mathcal{C}_{\mathrm{c}}$ smaller and wider than $\mathcal{C}$ (Caccia et al. 1987). As will be illustrated in Sect. 2.2.2, these effects do not affect the determination of $\boldsymbol{V}(h)$, as the only information needed is the position of each correlation peak. An example of a measured cross-correlation is given in Fig. 1. Three triplets can easily be identified, corresponding to altitudes of 2400, 6500 and $19500 \mathrm{~m}$ above sea level.

\subsubsection{Experimental method}

Our GS instrument measures simultaneously the autocorrelation $C^{* *}(\boldsymbol{r})$ of the scintillation images (of 1 to $2 \mathrm{~ms}$ exposure time), and two cross-correlations, $C_{\mathrm{c}}^{* *}(\boldsymbol{r}, 20 \mathrm{~ms})$ and $C_{\mathrm{c}}^{* *}(\boldsymbol{r}, 40 \mathrm{~ms})$, of images separated by a lapse $\Delta t=$ $20 \mathrm{~ms}$ and $\Delta t=40 \mathrm{~ms}$. The number of images used for these calculations varies from 1000 to 2000, depending on the star magnitudes, and the computations are made in quasi-real time, using a Matrox Genesis board equipped with a C80 Digital Signal Processor. One set of correlations is obtained every 45 to $90 \mathrm{~s}$, depending on the number of images processed. Each detector pixel covers a square area on the pupil, of side $\mathrm{d} x=2.8 \mathrm{~cm}$ (although this value can vary for different telescopes). The consequent eventual spatial filtering is taken into account in the maximum entropy algorithm used for calculating $C_{\mathrm{N}}^{2}(h)$.

An interactive algorithm has been developed for the determination of $\boldsymbol{V}, h$ and the turbulence intensity of the different turbulent layers. The input data are the crosscorrrelations $C_{\mathrm{c}}^{* *}(\boldsymbol{r}, 20 \mathrm{~ms})$ and $C_{\mathrm{c}}^{* *}(\boldsymbol{r}, 40 \mathrm{~ms})$, as well as the corresponding $C_{\mathrm{N}}^{2}(h)$ calculated from the autocorrelation using the method presented in Sect. 2.1. For a given triplet (as in Fig. 1), the user identifies the position of the central peak and that of either of the lateral peaks. The central peak position gives $\boldsymbol{V}$, and the distance between the central and the lateral peaks gives a preliminary

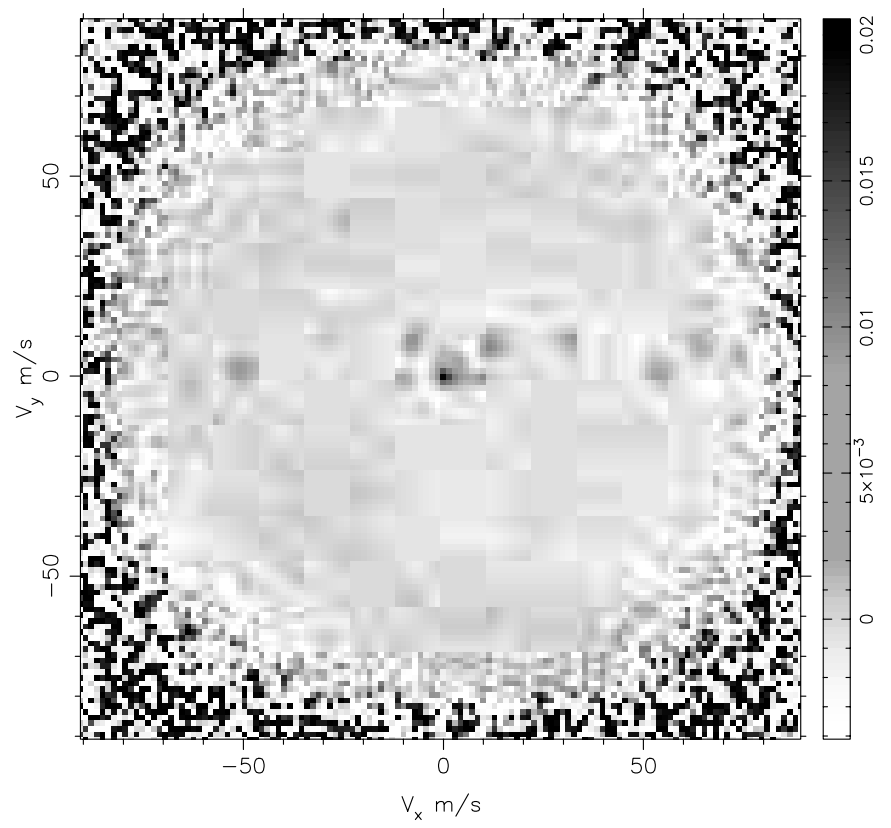

Fig. 1. Cross-correlation of scintillation images separated by $\Delta t=20 \mathrm{~ms}$, obtained with the double star $\kappa$ Pupis, of angular separation $\rho=9.9$ arcsec. The exposure time was $1 \mathrm{~ms}$. The analysis plane was situated at $h_{\mathrm{gs}}=-3 \mathrm{~km}$. Each turbulent layer produces 3 peaks aligned along the horizontal axis. Three triplets can be seen. The coordinates of the central peak of each of the 3 triplets give the wind velocity of the corresponding layer

altitude $h_{\mathrm{p}}$. The turbulence intensity associated with that triplet, and the final altitude, are found from the $C_{\mathrm{N}}^{2}(h)$ profile, as follows: the triplet is associated an uncertainty $\delta h_{\mathrm{p}}$ of the altitude determination, which depends on the altitude resolution $\Delta H$, given by Eq. (5), an estimated smearing of the peaks due to wind velocity fluctuations, the pixel size, and the peak amplitudes. The actual altitude $h$ selected for the layer is that corresponding to the maximum $C_{\mathrm{N}}^{2}$ value between $h_{\mathrm{p}}-\delta h_{\mathrm{p}} / 2$ and $h_{\mathrm{p}}+\delta h_{\mathrm{p}} / 2$. Finally, the turbulence intensity $I_{C_{\mathrm{N}}^{2}}$ associated with this layer is defined to be equal to the integral of the $C_{\mathrm{N}}^{2}(h)$ profile between the altitudes $h-\Delta H / 2$ and $h+\Delta H / 2$. Once a triplet has been analyzed, it is eliminated from the cross-correlations by subtracting a theoretical triplet calculated using the retrieved parameters $\boldsymbol{V}, h$, and $I_{C_{\mathrm{N}}^{2}}$.

Often there are several layers apparently at the same altitude, with different velocities. Our interpretation is that they are actually situated at slightly different altitudes, which cannot be resolved in the $C_{\mathrm{N}}^{2}(h)$ profile. The measured $C_{\mathrm{N}}^{2}$ at the altitude of these layers is in fact the sum of the real $C_{\mathrm{N}}^{2}$ of each layer. From this measured $C_{\mathrm{N}}^{2}$, a turbulence intensity $I_{C_{\mathrm{N}}^{2}}$ is calculated as explained above, and it is distributed to the layers at the same altitude, with weights proportional to the peak amplitudes in each of the corresponding triplets, to obtain the final $I_{C_{\mathrm{N}}^{2}}$ values for each layer.

All the analysis presented so far in this section has been developed for observations at the zenith. When the 


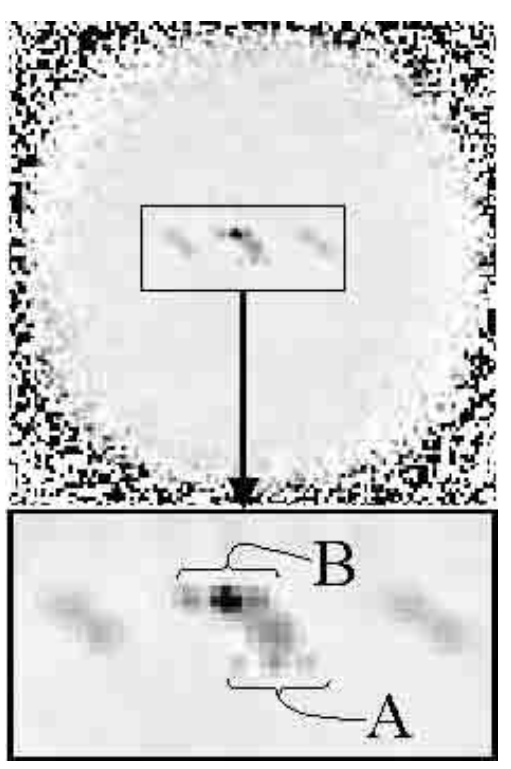

Fig. 2. Cross-correlation of scintillation images separated by $\Delta t=20 \mathrm{~ms}$, with the analysis plane at $h_{\mathrm{gs}}=-3 \mathrm{~km}$. Triplets $\mathrm{A}$ and $\mathrm{B}$ correspond to turbulence inside and outside the telescope dome, respectively

source has a zenith angle $z, h$ and $h_{\mathrm{gs}}$ must be replaced by $h \sec (z)$ and $h_{\mathrm{gs}} \sec (z)$. The altitude axis in each turbulence profile and wind profile is corrected by the factor $\sec (z)$, by $h_{\mathrm{gs}}$, and by the altitude of the observatory, to obtain the altitude above sea level.

The wind velocity vector $\boldsymbol{V}$, obtained with the method explained above, is in fact the projection of the actual horizontal wind vector on the wavefront plane. So far, we have not included the de-projection in our algorithm, because it has little consequence on the results, as the observations were carried out at small zenith angles $\left(z<30^{\circ}\right)$. The direction of $\boldsymbol{V}$ is strongly affected by the alignment of the $X$ axis of the detector pixels with the position angle of the double star, carried out before the observations. To obtain $\boldsymbol{V}$ in the cardinal reference system, a correction must be applied, which can be done in a post-processing phase. For the calculation of the temporal parameters relevant for AO, the orientation of $\boldsymbol{V}$ is not important.

\subsubsection{Dome seeing detection}

Inside the telescope dome, the mean wind velocity is zero. Thus, the turbulence inside the dome will produce a triplet located at the center of the cross-correlations, like triplet $\mathrm{A}$ in Fig. 2. However, we cannot be sure that the central triplet corresponds exclusively to the dome turbulence, unless we detect at least another triplet at the same altitude (that of the observatory) and with a non-zero velocity, like triplet B in Fig. 2. In this case, the triplet(s) with non-zero velocity is (are) associated with turbulence close to the ground but outside the dome, and the triplet with zero velocity (or less than the velocity resolution $\mathrm{d} V=\mathrm{d} x / \Delta t)$ is associated with dome turbulence.

The algorithm gives one of three possible attributes to the dome seeing detection:

- Detected: One layer with $V \leq \mathrm{d} V$ and $h=0 \pm \Delta H / 2$, and at least one layer with $V>\mathrm{d} V$ and $h=0 \pm \Delta H / 2$ were detected;
- Ambiguous: One layer with $V \leq \mathrm{d} V$ and $h=0 \pm \Delta H / 2$, but no layer with $V>\mathrm{d} V$ and $h=0 \pm \Delta H / 2$ were detected;

- Not detected: No layer with $V \leq \mathrm{d} V$ and $h=0 \pm \Delta H / 2$ was detected.

If the dome seeing is detected, the values of $I_{C_{\mathrm{N}}^{2}}$ associated with the turbulence inside and outside the dome are calculated as in the case, explained above, of several turbulent layers at apparently the same altitude. The only difference is an additional correction, which accounts for the slower temporal decorrelation of the turbulence inside the dome than that from outside.

\subsubsection{New $C_{N}^{2}(h)$ processing excluding dome seeing}

Let us imagine that dome seeing has been detected using the method in Sect. 2.2.3 and let us define its contribution to scintillation through the knowledge of the kernel, as expressed in Eq. (4):

$$
\begin{aligned}
B_{\text {Dome-gs }}^{* *}(x) & \equiv C_{\|}^{* *}-C_{\perp}^{* *} \\
& =\int \mathrm{d} h K(x, h)\left(C_{\mathrm{N}}^{2} \Delta h\right)_{\text {Dome }} \delta(h),
\end{aligned}
$$

where we have written $\left(C_{\mathrm{N}}^{2} \Delta h\right)_{\text {Dome }}$ instead of the turbulence intensity $I_{C_{\mathrm{N}}^{2}}$ in the dome, to maintain the analogy with Eq. (4), and $\delta(h)$ restrains the integral to $h=0$, the altitude of the observatory. In other words, the scintillation due to dome seeing is given by the line in the kernel that coincides with the observatory altitude, weighted with the optical turbulence which is derived from the previous multiple layer detection. Then, instead of processing Eq. (4), we process the difference $B_{\text {gs }}^{* *}-B_{\text {Dome-gs }}^{* *}$, using exactly the same maximum entropy method.

When the detection of the dome seeing is ambiguous, as explained above, there are two extreme options for the value given to $\left(C_{\mathrm{N}}^{2} \Delta h\right)_{\text {Dome }}$ : to associate the ambiguous triplet exclusively to turbulence inside the dome or exclusively to turbulence outside the dome. In the first case, $\left(C_{\mathrm{N}}^{2} \Delta h\right)_{\text {Dome }}$ takes the value of the $I_{C_{\mathrm{N}}^{2}}$ associated with the ambiguous triplet, and in the second case, $\left(C_{\mathrm{N}}^{2} \Delta h\right)_{\text {Dome }}$ takes the value of zero.

We have performed 3 different data reductions for the calculation of $C_{\mathrm{N}}^{2}(h)$ :

- Case N ("Normal"): without removing dome turbulence. This is exactly the data reduction that was performed before the development of the algorithm for the dome seeing detection;

- Case D ("Dome"): removing dome turbulence, and in case of ambiguity, the turbulence intensity corresponding to the ambiguous triplet is entirely associated with the dome, thus it is removed;

- Case A ("Atmosphere"): removing dome turbulence, and in case of ambiguity, the turbulence intensity corresponding to the ambiguous triplet is entirely associated with a layer outside the dome, thus it is not removed. 


\section{Observational campaign}

In 1998, a site testing campaign (Vernin et al. 2000) took place at Cerro Tololo and Cerro Pachón, Chile, in the framework of the Gemini program to implement an AO system at the $8.1 \mathrm{~m}$ Gemini South Telescope. The campaign was performed under the responsibility of Jean Vernin by the Département d'Astrophysique of the NiceSophia Antipolis University, after being selected from a request of proposals issued by the Gemini Observatory.

Several instruments were deployed. Our GS was used to monitor the $C_{\mathrm{N}}^{2}(h)$ and $\boldsymbol{V}(h)$ profiles. Instrumented balloons were launched to sound the fine vertical structure of the optical turbulence (by means of microfluctuation measurements of the temperature field) and other meteorological parameters, such as the wind velocity. A mast was equipped with the same kind of detectors as the ballons and used to measure the turbulence in the surface layer. The grating scale monitor, also called generalized seeing monitor (Martin et al. 1998; Avila et al. 1997b; Avila 1998; Conan et al. 2000b; Martin et al. 2000), provided measurements of the wavefront outer scale, the Fried parameter, and estimations of the wavefront velocity displacement, and the isoplanatic angle.

Four intensive campaigns were carried out during 1998: 12-18 January, 9-15 April, 14-20 July and 2-9 October. In the first three runs only the GS and the balloons were used, whereas in the last campaign all the instruments were used simultaneously. The GS was installed at the $1.5 \mathrm{~m}$ telescope of Cerro Tololo Interamerican Observatory (CTIO), the operational observatory nearest to Cerro Pachón. At Cerro Pachón the balloons were launched, and the other instruments were installed. The coordinates of Cerro Tololo are $\left(30^{\circ} 09^{\prime} 55^{\prime \prime} \mathrm{S}, 70^{\circ} 48^{\prime} 53^{\prime \prime} \mathrm{W}\right)$ and those of Cerro Pachón are $\left(30^{\circ} 14^{\prime} 26^{\prime \prime} \mathrm{S}, 70^{\circ} 44^{\prime} 12^{\prime \prime} \mathrm{W}\right)$, which corresponds to $11.5 \mathrm{~km}$ between both sites.

The instrumental setup of the GS was the same as that discribed by Avila et al. (1998). An independent scintillometer was attached to the telescope, and sampled by the computer hosting the Matrox board (see Sect. 2.2.2).

\section{Results}

\subsection{Wind profiles}

For the comparison of wind profiles obtained with the GS and the instrumented balloons we selected the GS data obtained approximately during the corresponding balloon ascent, and in the closest possible direction to the flight, which generally was towards the East. Figure 3 shows examples of comparison of measurements of $|\boldsymbol{V}(h)|$. The lines represent balloon profiles and the dots GS data, obtained on two different nights. The GS provided one velocity profile approximately every minute, leading to 76 and 50 profiles made of 341 and 221 individual velocity values, for the nights on January 13th (Fig. 3a) and 14th (Fig. 3b) 1998, respectively. Each instrumented balloon gives a wind speed value every $6 \mathrm{~m}$, approximately, along its ascending path, whereas the GS provides wind
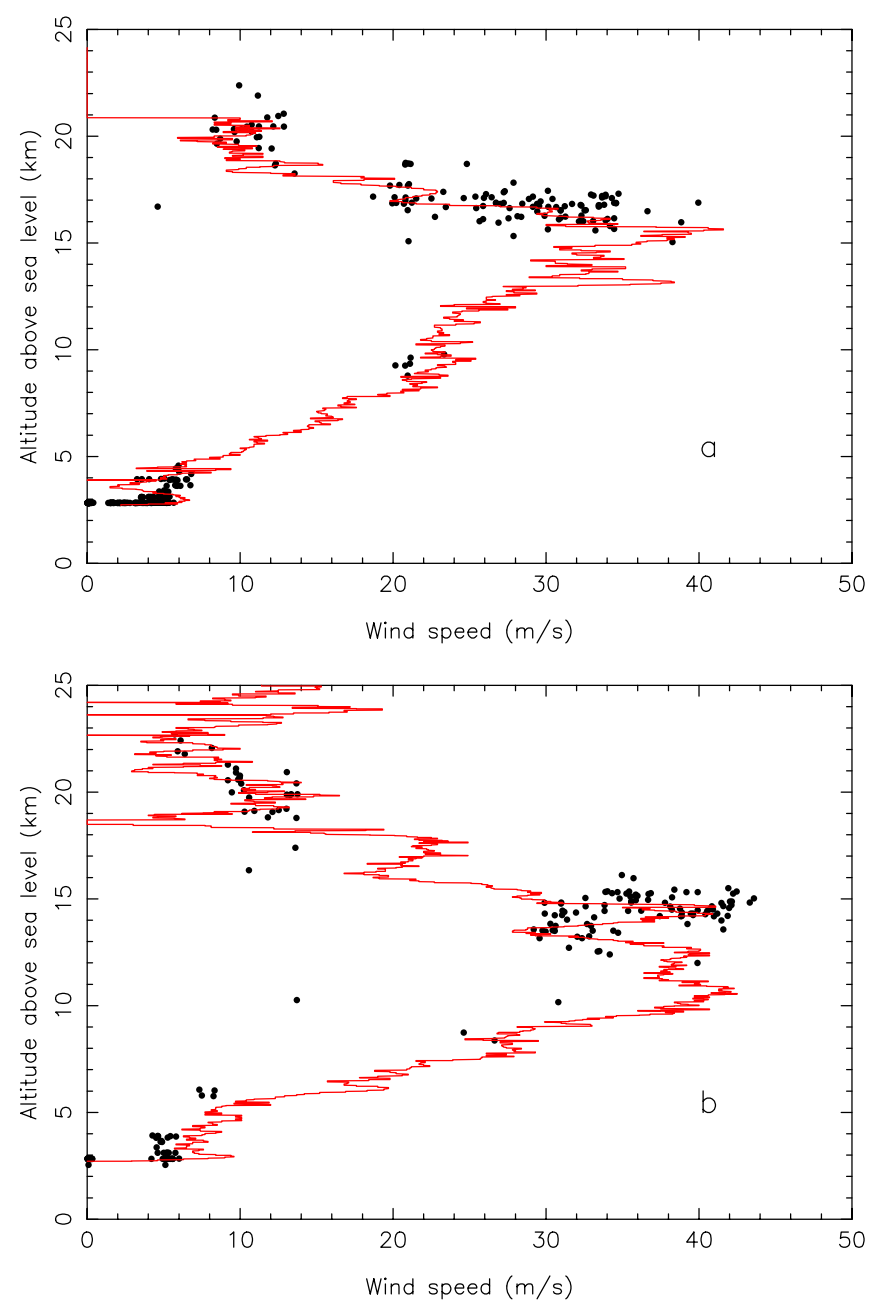

Fig. 3. Wind speed as a function of altitude above sea level for 2 different nights: a) January 13th, b) 14th 1998 UT. The line represents the measurements carried out in situ with instrumented balloons, and the dots are the measurements obtained with the GS, during the corresponding balloon ascent. The lowest altitude where data are plotted corresponds to the observatory altitude

speed values only where optical turbulence is detected. This explains the concentration of GS speed values at some altitudes. A remarkable correspondence is noticed between the GS and balloon results. For a comparison of the wind directions measured with each instrument, we need the Position Angle (PA) of the double star observed. The position of each star in the double system $\kappa$ Pupis $\left(\alpha_{1950}=073727.1, \delta_{1950}=-264814\right.$ and $\left.\alpha_{1950}=073726.90, \delta_{1950}=-264810.8\right)$, used for the GS observation in this comparison, lead to a PA of $40^{\circ}$. Using this estimated PA, and a necessary symmetry relative to the rows of the CCD, we calculated the quantity

$\theta=\left(360-\theta_{\mathrm{gs}}\right)-40$,

where $\theta_{\mathrm{gs}}$ is the direction of the wind velocity measured by the GS, with respect to the rows of the CCD. In Fig. 4, the solid lines represent the wind PA measured by the balloons and the dots indicate $\theta$, for two nights. A good 

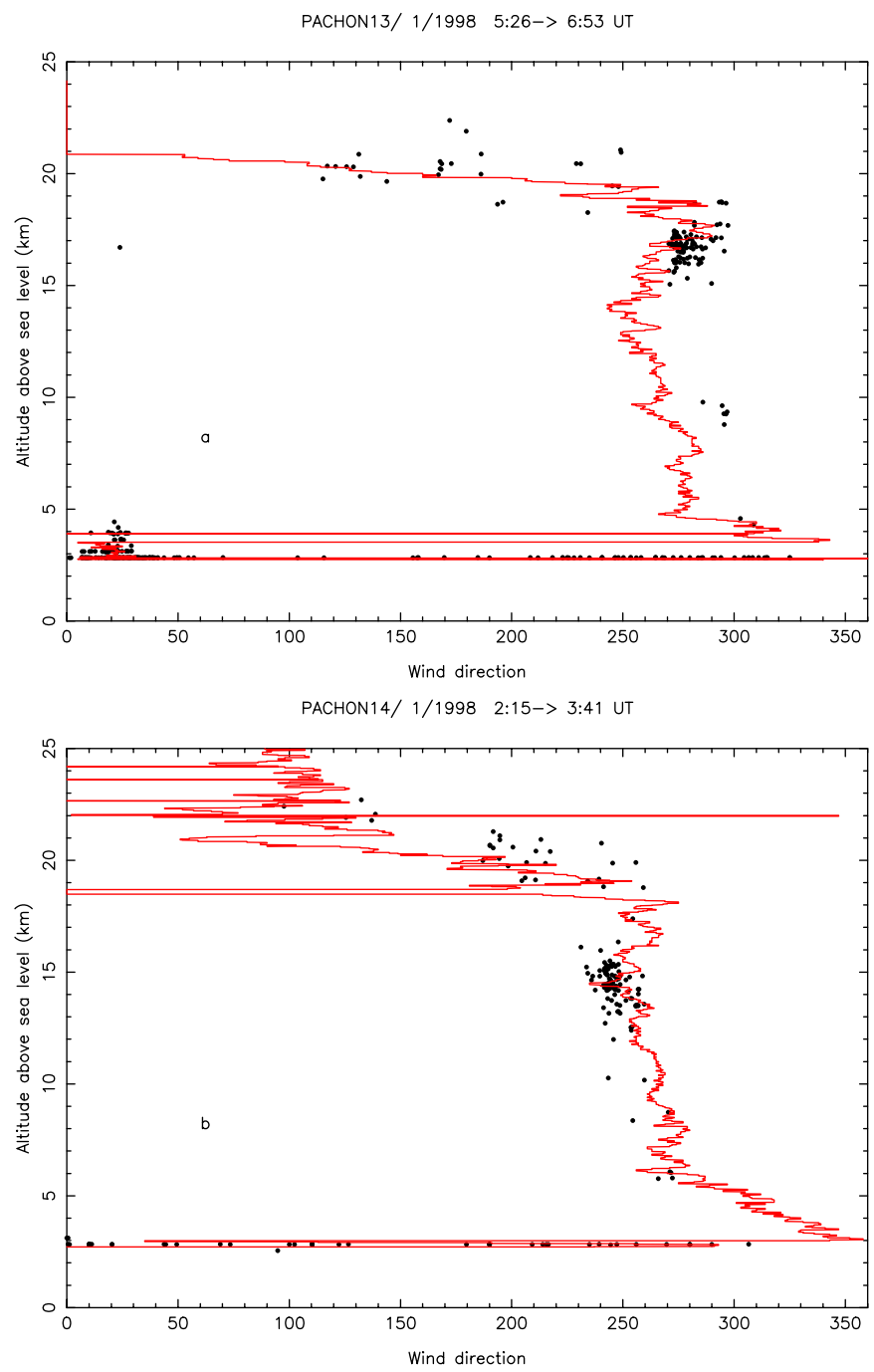

Fig. 4. Wind direction as a function of altitude above sea level for the same conditions as in Fig. 3

correspondence is found in both nights. For other nights the agreement is equivalent or better.

The GS velocity values seem to be concentrated in zones where the balloon data show strong vertical gradients of the wind velocity (either in its modulus or its direction, shown in Figs. 3 and 4, respectively), which agrees with the scenario in which the velocity gradient breaks the stratification, generating dynamical turbulence, which in turn, in the presence of a potential temperature gradient, generates optical turbulence (Coulman et al. 1995; Avila \& Vernin 1998; Werne \& Fritts 1999, and references therein).

The spread of the GS data can be attributed to variations of the wind velocity and to measurement uncertainties and errors, which are indistiguishable. Nevertheless, a statistical study of the difference between GS and balloon speed measurements was performed from 1461 GS speed values in 628 profiles obtained during the 8 selected nights. Two approaches were followed. We first compute simply the difference (in absolute value) of each GS speed measurement and the balloon value obtained at the

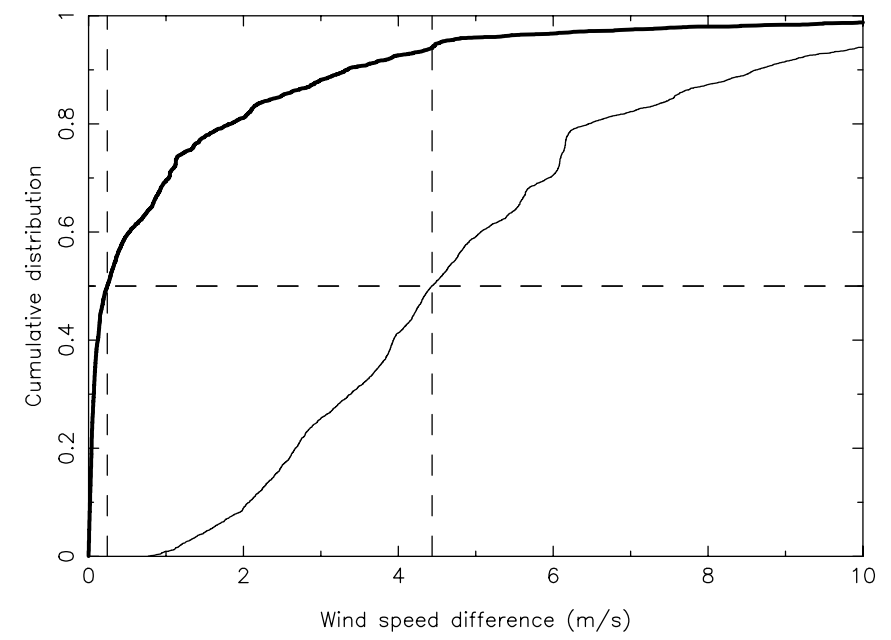

Fig. 5. Cumulative distributions of $\Delta V_{\mathrm{d}}$ (thin line) and $\Delta V_{\min }$ (bold line). The dashed lines indicate the median values

altitude corresponding to the GS data point. This direct difference $\Delta V_{\mathrm{d}}$ can result from velocity uncertainties, but also from the uncertainty in the altitude of the GS data point, which is known from Eq. (5). The second approach for the comparison of the GS and balloon measurements attempts to separate the influence of the altitude uncertainty. For each GS speed measurement, we determine the minimal difference $\Delta V_{\min }$ of the GS value and the balloon measurements that fall within the altitude uncertainty of the GS data point. The cumulative distributions of $\Delta V_{\mathrm{d}}$ and $\Delta V_{\text {min }}$ are plotted in Fig. 5 by the thin and bold lines, showing median values of 4.42 and $0.24 \mathrm{~m} / \mathrm{s}$, respectively. This means that in $50 \%$ of the cases, the wind speed measured by the GS at a given altitude differs less than $4.42 \mathrm{~m} / \mathrm{s}$ from the balloon measurement at exactly the same altitude. In $50 \%$ of the cases there exists a balloon value within the GS altitude uncertainty which differs less than $0.24 \mathrm{~m} / \mathrm{s}$ from the GS speed measurement.

An example of the wind speed as a function of time and altitude is shown in Fig. 6. Five layers are clearly distinguished at approximately 2.2, 4.5, 6.5, 12 and $18 \mathrm{~km}$, and a less defined layer is found at $17 \mathrm{~km}$. The fastest and the slowest layers are found at $12 \mathrm{~km}$, and at the observatory altitude $(2.2 \mathrm{~km})$, respectively.

\subsection{Dome seeing detection}

In Fig. 7 we show the dome seeing and the seeing near the ground (NG seeing) calculated from the integral of $C_{\mathrm{N}}^{2}(h)$ over the first kilometer, as a function of time, for processing cases $\mathbf{D}$ and $\mathbf{A}$ defined in Sect. 2.2.4. NG seeing does not include dome seeing. Only a short period of one night is shown, where the effect of the two processing cases is clearly seen. During that period, four ambiguous detections of the dome seeing were registered, and these cause the peaked structures in the processing case $\mathbf{A}$ (Fig. $7 \mathrm{~b}$ ): The turbulence intensity of the ambiguous triplet is entirely associated with the atmosphere outside the dome, 


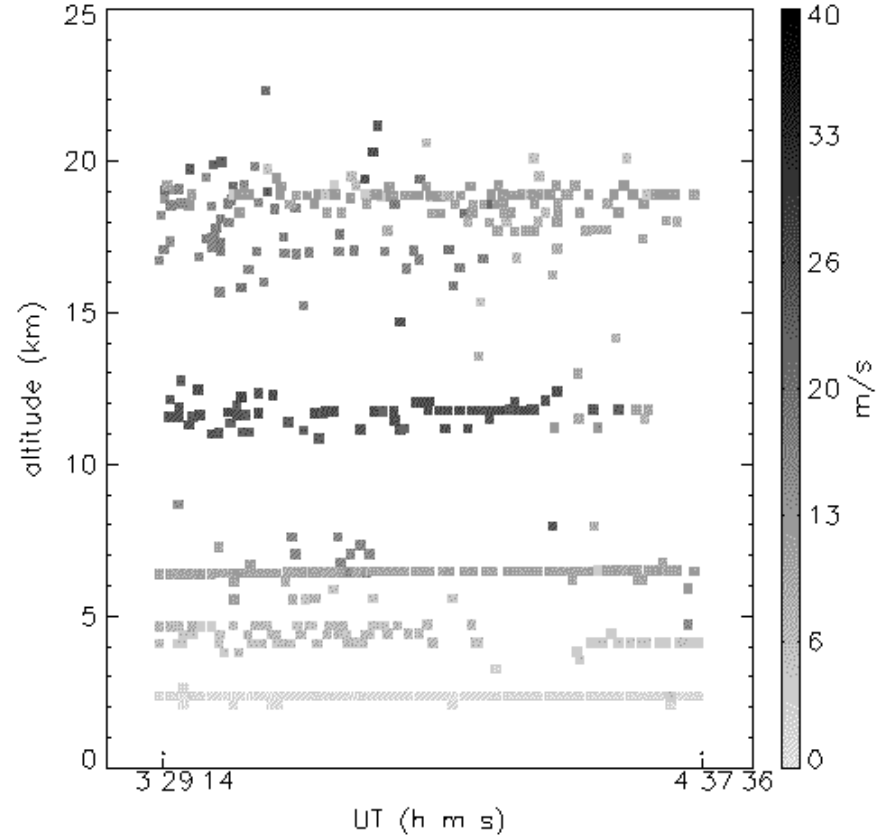

Fig. 6. Wind speed as a funtion of altitude above sea level (vertical axis) and time (horizontal axis) obtained with the GS, in part of the night of January 19th 1998 UT. The gray scale indicates the wind speed

provoking a sudden increase of NG seeing and a sudden decrease of dome seeing. It is unlikely that such drastic variations are real. In the $\mathbf{D}$ case (Fig. 7a), there are no signs of the ambiguous detection of dome seeing, which indicates that most probably the ambiguous triplet corresponds to turbulence inside the dome. However, in general, one expects that $\mathbf{D}$ processing tends to overestimate the dome seeing, and $\mathbf{A}$ processing to underestimate it.

\section{3. $C_{N}^{2}(h)$ profiles}

Figure 8 shows a typical example of $C_{\mathrm{N}}^{2}(h, t)$ obtained during one night, with $\mathbf{D}$ processing. The temporal sampling is one profile every $50 \mathrm{~s}$. The double stars observed that night were $\theta$ Eri $\left(\rho=8.3^{\prime \prime}\right.$, visible magnitudes $m_{1}=3.4$ and $\left.m_{2}=4.5\right), \kappa \operatorname{Pup}\left(\rho=9.9^{\prime \prime}, m_{1}=4.5\right.$ and $\left.m_{2}=4.8\right)$ and $\alpha \operatorname{Cru}\left(\rho=4^{\prime \prime}, m_{1}=1.3\right.$ and $\left.m_{2}=1.8\right)$. The analysis plane was located at $h_{\mathrm{gs}}=-3 \mathrm{~km}$. The $C_{\mathrm{N}}^{2}(h)$ profiles between 3 and 5 UT were obtained with the same data as the $|\boldsymbol{V}(h)|$ profiles shown in Fig. 6. Thus, the same layers can be identified (see Sect. 4.1). The turbulence at $6.5 \mathrm{~km}$ is extremely intense. It is even more intense than that near the ground, which is not a common feature. The strong turbulence that appears around 4h30 UT, at 18 $\mathrm{km}$, was encountered occasionally, mainly during the winter run (July 98). $C_{\mathrm{N}}^{2}$ values at altitudes within $\sim 1 \mathrm{~km}$ of the ground must be understood as part of the response of the turbulence at ground level. At lower altitudes, nonzero values are produced by the inversion process, which is very sensitive to noise in that range because the signal (i.e. the scintillation variance) is proportional to $\left(h-h_{\mathrm{gs}}\right)^{5 / 6}$.
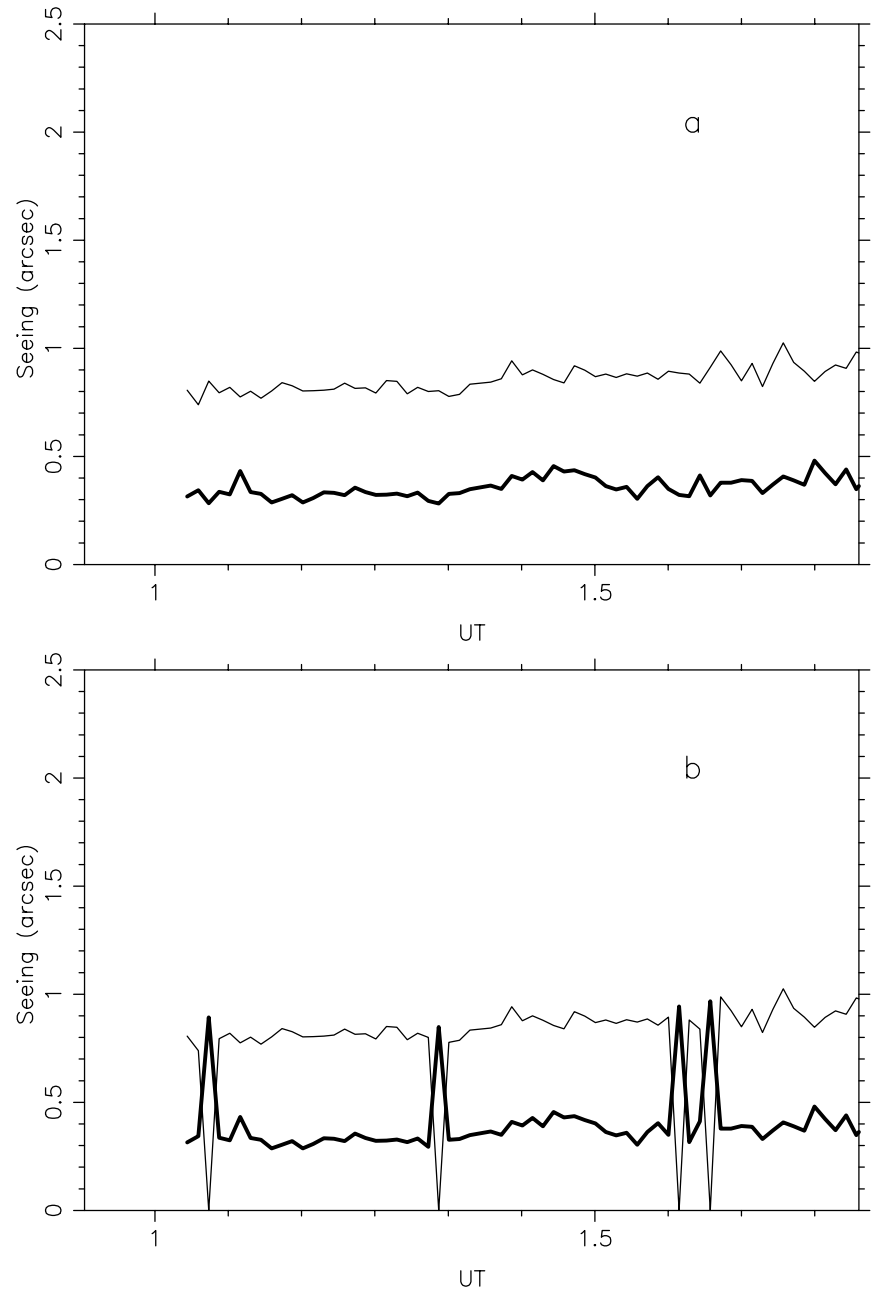

Fig. 7. Dome seeing (thin line) and NG seeing (thick line) for a short period of January 18th 1998, using processing cases D a) and $\mathbf{A} \mathbf{b}$ ) (see text). The ambiguous detections of the dome seeing cause the peaked features obtained with processing case A. The seeing values were calculated at a wavelength $\lambda=$ $0.5 \mu \mathrm{m}$

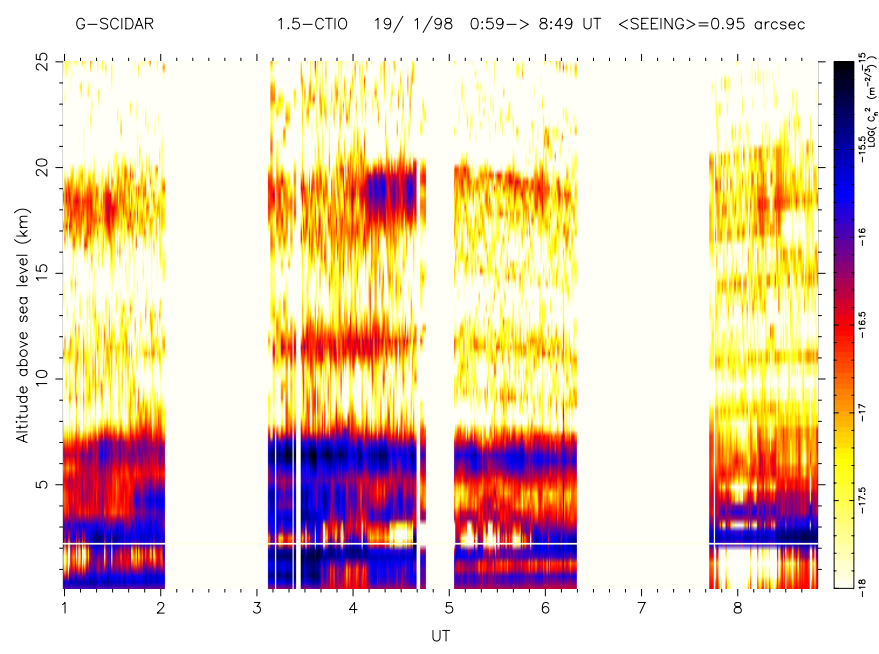

Fig. 8. $C_{\mathrm{N}}^{2}$ as a function of time and altitude, obtained on January 19th 1998 (UT). The grey level represents $\log \left(C_{\mathrm{N}}^{2}\right)$ as coded by the scale on the right. The horizontal line at $2.2 \mathrm{~km}$ represents the observatory altitude 


\section{Discussion and conclusion}

A method for monitoring simultaneously $C_{\mathrm{N}}^{2}(h)$ and $\boldsymbol{V}(h)$ has been presented. The innovative aspects are the wind profiles and the dome seeing measurements. Comparison of wind velocity profiles obtained with GS and instrumented balloons demonstrated the effectiveness of the method. A study of the turbulence conditions at Cerro Pachón was carried out in four one-week runs uniformly distributed during 1998, leading to a statistical analysis of the parameters relevant for the development of the AO system at Gemini South Telescope. These parameters will be presented in a forthcoming paper.

The capability of monitoring $C_{\mathrm{N}}^{2}(h)$ and $\boldsymbol{V}(h)$ can have very positive consequences for the optimization of currently operational AO systems and the development of future innovative systems. For current AO systems, the knowledge of the $C_{\mathrm{N}}^{2}$ profile, together with wavefront sensor residual errors, the control servo gains, and the calibration of any unsensed static aberration can lead to the calculation of the point spread function across the fieldof-view (Véran et al. 1997). If additionally the $|\boldsymbol{V}(h)|$ profile is known, the temporal decorrelation of the PSF can be calculated. The isoplanatic angle of an AO system with a single deformable mirror (DM) can be increased if the DM is conjugated at an optimal altitude (Racine \& Ellerbroek 1995; Herriot et al. 1998). Multi-conjugate AO systems with several correcting elements, each conjugated to different layers, can provide a non-variant PSF over a corrected field-of-view of 1 to 2 arcmin (Fusco et al. 1999). For the development of such sytems at a given site, a statistical study of $C_{\mathrm{N}}^{2}(h)$ is essential in order to estimate the optimal conjugation altitudes, and the velocity profiles would indicate the temporal bandwidth necessary for each correcting element. Tomographic methods, using multiple guide stars, have been proposed to extend AO correction to the whole sky (Tallon \& Foy 1990). An experimental demonstration has recently been published by Ragazzoni et al. (2000), who stated that the tomographic matrix used depends upon $C_{\mathrm{N}}^{2}(h)$. Kaiser et al. (2000) proposed a new strategy for deep wide-field high-resolution imaging, in which $C_{\mathrm{N}}^{2}$ and $\boldsymbol{V}$ profiles are necessary for the determination of a matrix that must be inverted. Andersen (1998) proposed a method for sensing the wavefront in a wide field. He claims that the interpretation of the data he obtained would benefit from GS measurements.

However, it is still hard to imagine a system which could correct for extreme turbulence conditions, which can occasionally be encountered at any astronomical site. Under such conditions, observing time would be more effectively spent on science programs with less demands on the delivered image quality. Monitoring turbulence and wind profiles could warn in real time about such extreme conditions, but it would be more desirable to be able to forecast them (Masciadri et al. 1997, 1999a,b), as well as the optimal conditions, and establish a flexible-scheduling of the science programs.
The monitoring of turbulence and wind profiles with GS, demonstrated in this paper, could be exploited for the most ambitious applications cited above only if an automatic version of the algorithm for the wind velocity determination is developed. The use of a CLEAN algorithm for that purpose has already been tried by Klückers et al. (1998).

Acknowledgements. We are indebted to M. Azouit and A. Agabi for taking care of the balloon data, and many technical aspects. A special mention is due to M. Chun for his participation in the observations, and fruitful discussions about the the impact of GS in AO system developments. We acknowledge E. Masciadri for participating in the GS data acquisition and useful suggestions about GS versus balloon data comparison. We are grateful to C. Dainty for his kind agreement of our citing of a private communication with his group. We also thank the CTIO and Gemini staff for their help during the observing campaigns. Funding for the site characterization campaign was provided by Gemini Observatory/AURA. Part of this work was developed under the grants number J32412E from CONACyT, and IN118199 from DGAPA-UNAM.

\section{References}

Andersen, M. I. 1998, New Astron. Rev., 42, 455

Avila, R. 1998, Ph.D. Thesis, Université de Nice-Sophia Antipolis

Avila, R., \& Vernin, J. 1998, in Interstellar Turbulence, ed. J. Franco, \& A. Carramiñana (Cambridge University Press) Avila, R., Vernin, J., \& Cuevas, S. 1998, PASP, 110, 1106

Avila, R., Vernin, J., \& Masciadri, E. 1997a, Appl. Opt., 36, 7898

Avila, R., Ziad, A., Borgnino, J., et al. 1997b, J. Opt. Soc. Am. A, 14,3070

Caccia, J., Azouit, M., \& Vernin, J. 1987, Appl. Opt., 26, 1288

Conan, R., Borgnino, J., Ziad, A., \& Martin, F. 2000a, J. Opt. Soc. Am. A, 17, accepted

Conan, R., Ziad, A., Borgnino, J., Martin, F., \& Tokovinin, A. 2000b, in Interferometry in Optical Astronomy, ed. P. J. Lena, \& A. Quirrenbach, SPIE, 4006

Coulman, C. E., Vernin, J., \& Fuchs, A. 1995, Appl. Opt., 34, 5461

Fuchs, A. 1995, Ph.D. Thesis, Université de Nice-Sophia Antipolis

Fuchs, A., Tallon, M., \& Vernin, J. 1994, in Atmospheric Propagation and Remote Sensing III, ed. W. A. Flood, \& W. B. Miller, 2222, 682

Fuchs, A., Tallon, M., \& Vernin, J. 1998, PASP, 110, 86

Fusco, T., Conan, J.-M., Michau, V., Mugnier, L. M., \& Rousset, G. 1999, in Propagation and Imaging through the Atmosphere III, ed. M. C. Roggemann, \& L. R. Bissonnette, SPIE, 3763, 125

Gendron, E., \& Lena, P. 1996, Ap\&SS, 239, 221

Herriot, G., Morris, S., Roberts, S., et al. 1998, in Adaptive Optical System Technologies, ed. D. Bonnaccino, \& R. K. Tyson, SPIE, 3353, 488

Kaiser, N., Tonry, J. L., \& Luppino, G. A. 2000, PASP, 112, 768

Klückers, V. A., Wooder, N. L., Adcock, M. A., Monro, I., \& Dainty, J. C. 1998, A\&AS, 130, 141

Martin, F., Conan, R., Tokovinin, A., et al. 2000, A\&AS, 144, 39 
Martin, F., Tokovinin, A., Ziad, A., et al. 1998, A\&A, 336, L49

Masciadri, E., Vernin, J., \& Bougeault, P. 1997, in The Three Galileos: the Man, the Spacecraft, the Telescope, vol. 220 (Dordrecht Kluwer Academic Publishers), 389

Masciadri, E., Vernin, J., \& Bougeault, P. 1999a, A\&AS, 137,203

Masciadri, E., Vernin, J., \& Bougeault, P. 1999b, A\&AS, 137,185

Noel, T. 1997, Ph.D. Thesis, Université Paris VI

Racine, R., \& Ellerbroek, B. 1995, in Adaptive Optical Systems and Applications, ed. R. Tyson, \& R. Fugate, SPIE, 2534, 248

Ragazzoni, R., Marchetti, E., \& Valente, G. 2000, Nature, 403, 54

Rocca, A., Roddier, F., \& Vernin, J. 1974, J. Opt. Soc. Am. A, 64, 1000

Roddier, F. 1981, in Progress in Optics, vol. XIX, ed. E. Wolf Schöck, M., \& Spillar, E. 1998, Opt. Lett., 23, 150

Schöck, M., \& Spillar, E. J. 1999, in Adaptive Optics Systems and Technology, ed. R. K. Tyson, \& R. Q. Fugate, SPIE, 3762
St-Jacques, D., \& Baldwin, J. E. 2000, in Interferometry in Optical Astronomy, ed. P. J. Lena, \& A. Quirrenbach, SPIE, 4006

Tallon, M. 1989, Ph.D. Thesis, Université de Nice-Sophia Antipolis

Tallon, M., \& Foy, R. 1990, A\&A, 235, 549

Tokovinin, A. A., Ziad, A., Martin, F., et al. 1998, in Adaptive Optics Systems and Technology, ed. D. Bonaccini, \& R. K. Tyson, SPIE, 3353, 1155

Véran, J.-P., Rigaut, F., Maître, H., \& Rouan, D. 1997, J. Opt. Soc. Am. A, 14, 3057

Vernin, J. 1992, in Wave propagation in Random Media (Scintillation) - Invited papers, ed. V. Tatarskii, A. Ishimaru, \& V. Zavorotny, SPIE (Press, Bellingham, Wash), 248

Vernin, J., Agabi, A., Avila, R., et al. 2000, 1998 Gemini site testing campaign Cerro Pachón and Cerro Tololo (AURA report)

Vernin, J., \& Azouit, M. 1983, J. Opt. (Paris), 14, 131

Werne, J., \& Fritts, D. 1999, Geophys. Res. Lett., 26, 439 